\title{
Anomalous behaviour of acoustooptic figure of merit under the conditions of collinear diffraction
}

\author{
Mys O., Kostyrko M., Vasylkiv Yu. and Vlokh R. \\ Vlokh Institute of Physical Optics, 23 Dragomanov Street, 79005 Lviv, Ukraine, \\ vlokh@ifo.lviv.ua
}

Received: 28.10 .2015

\begin{abstract}
Anisotropies of the acoustooptic figure of merit (AOFM) and the effective elastooptic coefficient under the conditions of collinear diffraction have been analyzed for $\mathrm{LiNbO}_{3}$ crystals. Narrow peak-like extremes have been revealed in the dependences of AOFM upon the orientation angle of the wave vector of the incident optical wave. We have also explained the reasons for the angular instability of the AOFM and the effective elastooptic coefficient.
\end{abstract}

Keywords: collinear acoustooptic diffraction, figure of merit, spatial anisotropy

PACS: $43.35 . \mathrm{Sx}$, 42.70.Mp

UDC: $535.012 .2+535.42+534.321 .9$

\section{Introduction}

A collinear acoustooptic (AO) diffraction is widely applied in different AO devices, e.g. tunable AO filters [1-4] and polarization converters [1,5]. In our earlier works [6, 7] we have shown that the collinear anisotropic AO diffraction is accompanied by abrupt changes in the AO figure of merit (AOFM). An anomalous dependence of the AOFM on the incident angle can be observed at the diffraction angles close to zero $(\gamma=0)$, manifesting itself in the appearance of very narrow peaks. Such peaks have already been revealed in the anisotropic crystals that belong to the systems of different point symmetry groups, at least in the trigonal and tetragonal systems represented by such well-known $\mathrm{AO}$ materials as $\mathrm{LiNbO}_{3}$ and $\mathrm{TeO}_{2}$ crystals [6, 7]. The appearance of such anomalies has been demonstrated in the work [8] while analyzing the behaviour of effective elastooptic coefficients (EECs). On the other hand, the appropriate anomaly does not appear under the conditions of collinear diffraction happening at $\gamma=180 \mathrm{deg}$, i.e. when one deals with a reflecting AO grating [6, 7]. Since the effect mentioned above makes the efficiency of the collinear diffraction sensitive to the propagation directions of the incident optical wave and the acoustic wave, the reasons for its appearance should be thoroughly clarified. This is the main aim of the present work.

\section{Results and discussion}

It is well known that the AOFM is described by the relation

$$
M_{2}=\frac{n_{i}^{3} n_{d}^{3} p_{e f}^{2}}{\rho v^{3}},
$$

where $n_{i}$ and $n_{d}$ are the refractive indices respectively of the incident and diffracted optical waves, $p_{e f}$ denotes the EEC, $v$ the acoustic wave velocity, and $\rho$ the material density. Let us analyze the AOFM anisotropy basing on the method developed in Ref. [7] for the trigonal crystals 
that belong to the symmetry groups $3 \mathrm{~m}, 32$ and $\overline{3} \mathrm{~m}$, and using $\mathrm{LiNbO}_{3}$ crystals as example. It would be suitable to recite the material parameters of lithium niobate (the point group $3 \mathrm{~m}$ ) needed in our further analysis. It is commonly accepted that one of the mirror symmetry planes in the $\mathrm{LiNbO}_{3}$ crystals is perpendicular to the crystallographic axis $a$ [9], while the $c$ axis is parallel to the three-fold symmetry axis. Here the crystallographic axes $a, b$ and $c$ correspond respectively to the $X, Y$ and $Z$ axes of Fresnel ellipsoid. The refractive indices are equal to $n_{o}=2.286$ and $n_{e}=2.203$ at the wavelength of optical radiation equal to $632.8 \mathrm{~nm}$ [9] (i.e., $n_{o}>n_{e}$ ). The elasto-optic coefficients of lithium niobate are as follows [10]: $p_{11}=-0.023 \pm 0.017$, $p_{12}=0.076 \pm 0.014, \quad p_{13}=0.147 \pm 0.019, \quad p_{31}=0.157 \pm 0.007, \quad p_{33}=0.141 \pm 0.013$, $p_{14}=0.057 \pm 0.004, p_{41}=-0.051 \pm 0.011$ and $p_{44}=0.126 \pm 0.004$ (at $\lambda=632.8 \mathrm{~nm}$ ). The elastic stiffness coefficients under the conditions of constant electric field are equal to $C_{11}=2.03$, $C_{12}=0.573, C_{13}=0.752, C_{33}=2.42, C_{44}=0.595, C_{66}=0.728$ and $C_{14}=0.085$ (in the units of $10^{11} \mathrm{~N} / \mathrm{m}^{2}$ ). Finally, the density of $\mathrm{LiNbO}_{3}$ amounts to $\rho=4640 \mathrm{~kg} / \mathrm{m}^{3}[11]$.

Now let us analyze the reasons for the peak-like extremes appearing in the dependences of AOFM and EEC on the $\theta+\gamma$ angle when the diffraction is collinear and all the three interacting waves propagate along the same direction. For simplicity, we analyze only a so-called type VII of AO interactions (see Refs. [6, 7]), which corresponds to the quasi-longitudinal acoustic wave propagating in the $X Z$ plane (see Fig. 1). Then the relations for the AOFM and the EEC are as follows:

$$
\begin{aligned}
& M_{2}^{(V I I)}=\frac{n_{o}^{3} n_{i}^{3}\left\{p_{e f}^{(V I I)}\right\}^{2}}{\rho\left[v_{Q L}(\chi)\right]^{3}}, \\
& p_{e f}^{(V I I)}=p_{14} \sin 2 \chi \sin \theta+p_{41} \cos ^{2} \chi \cos \theta,
\end{aligned}
$$

where $n_{i}=\frac{n_{o} n_{e}}{\sqrt{n_{o}^{2} \cos ^{2} \theta+n_{e}^{2} \sin ^{2} \theta}}, v_{Q L}(\chi)$ defines the dependence of the AW velocity on the orientation of the acoustic wave vector, and

$$
\chi=\arctan \left(\frac{n_{o} \sin (\theta+\gamma)-n_{o} n_{e} \frac{\sin \theta}{\sqrt{n_{o}^{2} \cos ^{2} \theta+n_{e}^{2} \sin ^{2} \theta}}}{n_{o} \cos (\theta+\gamma)-n_{o} n_{e} \frac{\cos \theta}{\sqrt{n_{o}^{2} \cos ^{2} \theta+n_{e}^{2} \sin ^{2} \theta}}}\right) .
$$

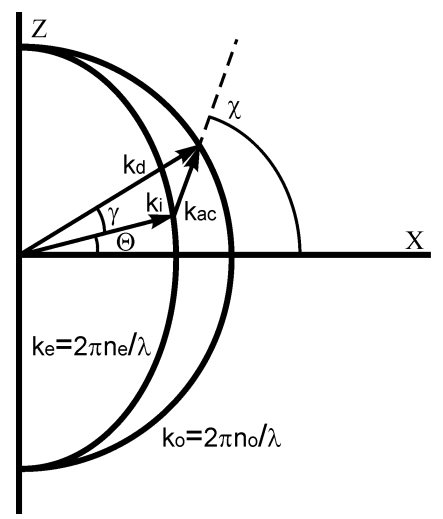

Fig. 1. Scheme of phase matching conditions corresponding to $\mathrm{AO}$ interaction in the $Z X$ plane: $k_{i}, k_{d}$ and $K_{a c}$ are the wave vectors of respectively the incident optical wave, the diffracted optical wave and the acoustical wave. 


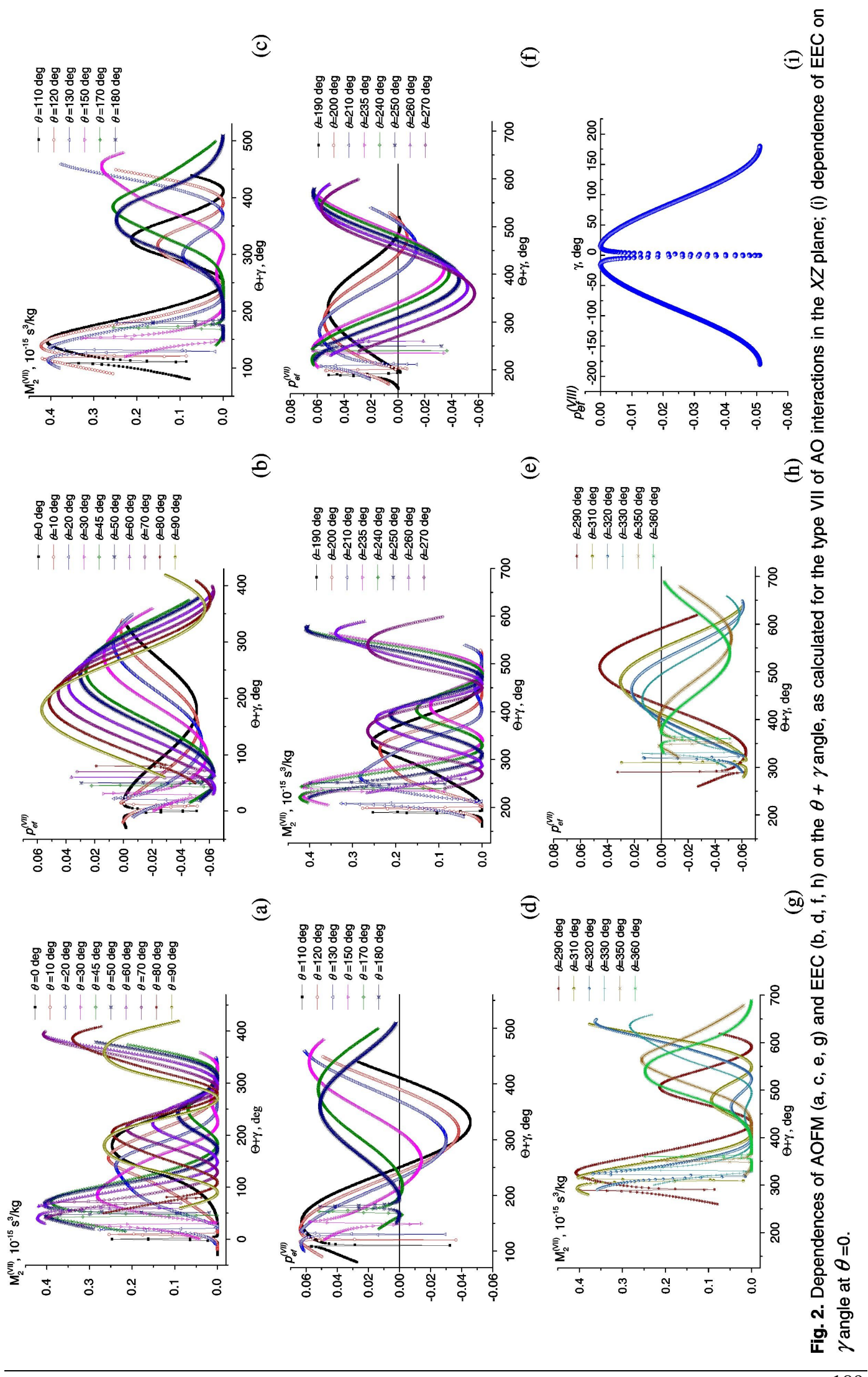

Ukr. J. Phys. Opt. 2015, Volume 16, Issue 4 
As readily seen from Fig. 2, the pronounced anisotropy and the peak-like extremes of the AOFM (Fig.2 a,c,e,g) are linked to appropriate anisotropy and the anomalies associated with the EEC (Fig.2 b,d,f,h). Then we are consider in detail the EEC anomalies that appear at different $\theta$ angles between the wave vector of the incident optical wave and the $X$ axis. The anomaly in the EEC at $\theta=0$ is characterized by a deep minimum, which is surrounded symmetrically by two maximums, corresponding to the condition $p_{e f}^{(V I I)}=0$ (Fig. 2 b,i). Increase in the $\theta$ angle (up to $\sim 45 \mathrm{deg}$ ) results in broadening of one of the maximums. At the same time the maximums become non-symmetric (Fig. 2 b). When we approach the value $\theta \approx 45 \mathrm{deg}$, the minimum is replaced by a sharp narrow maximum that increases when $\theta$ becomes $60 \mathrm{deg}$ with subsequent decrease. Notice that the collinear diffraction cannot exist at $\theta=90 \mathrm{deg}$, since this implies the direction of the optic axis. The dependence of the EEC on the $\theta+\gamma$ angle is reversed in the angular $\theta$ region from 90 to $180 \mathrm{deg}$ (Fig. $2 \mathrm{~d}$ ). The anomaly in the EEC at $\theta=180 \mathrm{deg}$ is characterized by a deep maximum, which is surrounded symmetrically by two minimums, corresponding to the condition $p_{e f}^{(V I I)}=0$. Finally, the dependence observed in the $\theta+\gamma$ interval ranging from 180 to $360 \mathrm{deg}$ becomes the same by the module of $p_{e f}$ as that seen in the region from 0 to $180 \mathrm{deg}$ (Fig. $2 \mathrm{f}, \mathrm{h}$ ).

For the case of collinear diffraction $(\gamma=0)$, the EEC is negative, being equal to $p_{e f}^{(V I I)}=p_{41}=-0.051 \pm 0.011$ at $\theta=0$, while at $\theta=60 \mathrm{deg}$ we have a positive $p_{e f}^{(V I I)}=\left(3 p_{14}+p_{41} / 2\right) / 4=0.036\left(p_{14}=0.057 \pm 0.004\right)[10]$. At $\theta=90 \mathrm{deg}$ we have $p_{e f}^{(V I I)}=0$. This is a reason why the EEC minimum observed in the vicinity of $\theta=0$ is replaced by a maximum detected when the $\theta$ angle approaches the value $\theta=60 \mathrm{deg}$. In fact, the EEC dependence on the $\theta$ angle observed under the conditions of collinear diffraction $(\gamma=0-$ see Fig. 3a) suggests that the maximal module of EEC value is reached at $\theta=0$ and $180 \mathrm{deg}$, while the minimum is seen in the vicinity of $\theta=34,90,146,214,270$ and $326 \mathrm{deg}$.
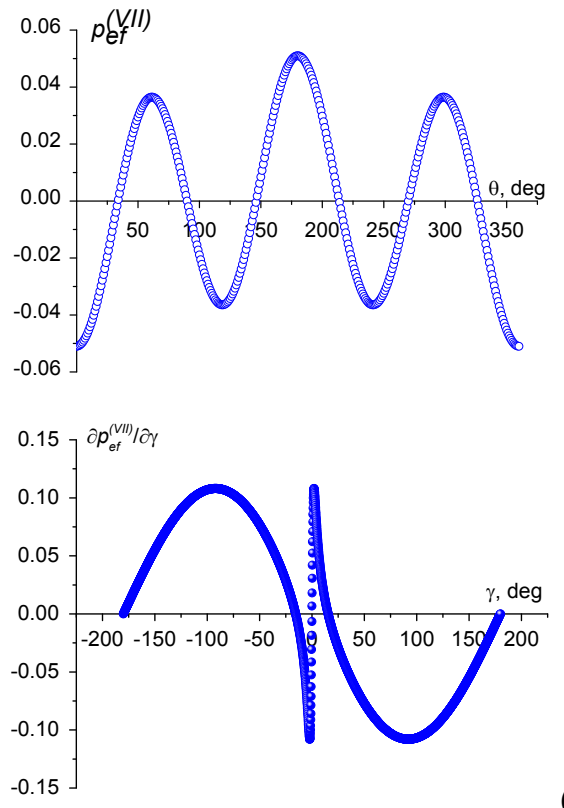

(a)

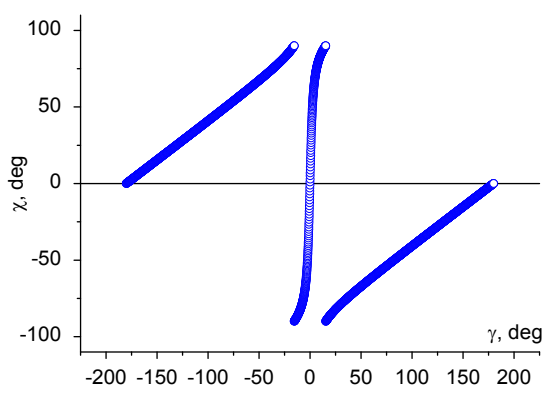

(b)

Fig. 3. Dependence of EEC on the $\theta$ angle under the exact conditions of collinear diffraction $(\gamma=0)$ (a), dependence of angle $\chi$ on $\gamma$ at $\theta=0$ (b) and dependence of $\partial p_{e f}^{(V I I)} / \partial \gamma$ on the $\gamma$ angle at 
Now let us consider the reasons for the angular instability of the EEC observed under the conditions close to those that correspond to the collinear diffraction. The quantities entering Eq. (3) are constitutive coefficients and functions of the geometric arguments, the angles $\theta$ and $\chi$. From Eq. (4) we have $\chi= \pm 90 \mathrm{deg}$ and $p_{e f}^{(V I I)}=0$ whenever the denominator is zero. The latter is reached under the condition

$$
\cos (\theta+\gamma)=\frac{n_{e} \cos \theta}{\sqrt{n_{o}^{2} \cos ^{2} \theta+n_{e}^{2} \sin ^{2} \theta}} .
$$

As seen from Fig. 2b,i at $\theta=0$, two zeros of $p_{e f}^{(V I I)}$ appear at the angular coordinates $\theta+\gamma= \pm 15 \mathrm{deg}$ (according to Eq. (5), we have $\operatorname{acos}(\theta+\gamma)=n_{e} / n_{o}=15.49 \mathrm{deg}$ ) which correspond to discontinuities of function $\left.\chi(\gamma)\right|_{\theta=0}$ (Fig. 3b) and zeroes values of function $\partial p_{e f}^{(V I I)} / \partial \gamma$ (Fig.3c). It is seen (Fig.3c) that collinear diffraction possess maximal instability at $\gamma= \pm 2.4 \mathrm{deg}$, which correspond to the positions of narrow extremums of function $\partial p_{e f}^{(V I I)} / \partial \gamma$. For all the other $\theta$ values, maximal $p_{e f}^{(V I I)}$ magnitudes are observed under condition (5). In other words, the efficiency of the collinear diffraction reveals an evident angular instability in the vicinity of $\gamma=0$. The reasons are as follows: (i) a strong dependence of the EEC on the incidence angle of the optical beam observed in the vicinity of $\gamma=0$, (ii) maximums of the module of EEC associated with orthogonality of the acoustic wave vector to the $X$ axis and (iii) a fact that the EEC should necessarily acquire a definite value at $\gamma=0$.

\section{Conclusions}

In the present work we have analyzed the anisotropies of both the AOFM and the EEC which appear under conditions of the collinear $\mathrm{AO}$ diffraction in the $\mathrm{LiNbO}_{3}$ crystals. The anomalies that manifest themselves as peak-like extremes have been revealed on the dependences of the AOFM on the orientation angle of the wave vector of the incident optical wave. The relevant conditions correspond to a close vicinity of the collinear diffraction. The narrow extremes are caused by the same anomalies appearing in the angular dependences of the EEC. In particular, we have found that the angular instabilities of the AOFM and the EEC at the collinear diffraction are caused by the following factors: (i) extremely strong dependence of the EEC on the incident angle of the optical beam in the vicinity of $\gamma=0$, (ii) EEC module maximums caused by orthogonality of the acoustic wave vector to the $X$ axis, and (iii) a definite value acquired by the EEC under the exact conditions of collinear diffraction.

\section{References}

1. Magdich L N and Molchanov V Ya, Acoustooptic devices and their application, New York: Gordon and Breach Science Pub. (1989).

2. Morasca S, Scarano D and Schmid S, Application of $\mathrm{LiNbO}_{3}$. Acoustooptic tunable switches and filters in WDM transmission networks at high bit rates. In: Book of Photonic Networks, Ed. by G Prati, Part 3. Advances in Optical Communications, pp. 458-472 (1997).

3. Nakazawa T, Taniguchi S and Seino M, 1999. Ti: $\mathrm{LiNbO}_{3}$ acousto-optic tunable filter (AOTF). FUJITSU Sci. Techn. J. 35: 107-112.

4. Fujii $\mathrm{Y}$ and Hayashi $\mathrm{H}, 1977$. Acousto-optic tunable filter using $\mathrm{LiNbO}_{3}$ crystals. Proc. SPIE. 99: $110-115$. 
5. Yudistira D, Janner D, Benchabane $\mathrm{S}$ and Pruneri V, 2009. Integrated acousto-optic polarization converter in a $\mathrm{ZX}$-cut $\mathrm{LiNbO}_{3}$ waveguide superlattice. Opt. Lett. 34: 3205-3207.

6. Mys O, Kostyrko M, Krupych O and Vlokh R, 2014. Anisotropy of acoustooptic figure of merit for $\mathrm{TeO}_{2}$ crystals. 2. Anisotropic diffraction. Ukr. J. Phys. Opt. 16: 38-60.

7. Mys O, Kostyrko M and Vlokh R, 2015. The anisotropy of acousto-optic figure of merit for $\mathrm{LiNbO}_{3}$ crystals: Anisotropic diffraction. Appl. Opt. (to be published).

8. Zyuryukin Yu A, Zavarin S V and Yulaev A N, 2009. Characteristic features of wideband anisotropic light diffraction in lithium-niobate crystal by a longitudinal acoustic wave. Opt. Spectrosc. 107: 152-156.

9. Weis R S and Gaylord T K, 1985. Lithium niobate: summary of physical properties and crystal structure. Appl. Phys. A. 37: 191-203.

10. Krupych O, Savaryn V and Vlokh R, 2014. Precise determination of full matrix of piezo-optic coefficients with a four-point bending technique: the example of lithium niobate crystals. Appl. Opt. 53: B1-B7.

11. Smith R T and Welsh F S, 1971. Temperature dependence of the elastic, piezoelectric, and dielectric constants of lithium tantalate and lithium niobate. J. Appl. Phys. 42: 2219-2230.

Mys O., Kostyrko M., Vasylkiv Yu. and Vlokh R. 2015. Anomalous behaviour of acoustooptic figure of merit under the conditions of collinear diffraction. Ukr.J.Phys.Opt. 16: 187-192.

Анотація. Проаналізовано анізотропію коефіцієнта акустооптичної якості і ефективного пружнооптичного коефіцієнта за умови колінеарної дифракції в кристалах $\mathrm{LiNbO}_{3}$. Виявлені пікоподібні екстремуми на залежностях коефіцієнта акустооптичної якості від кута орієнтації хвильового вектора падаючої оптичної хвилі, щзо відповідають кутовому околу колінеарної дифракції. 3'ясовано причину кутової нестабільності коефіцієнта акустооптичної якості і ефективного пружнооптичного коефіціснта. 